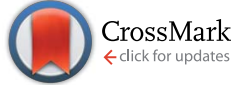

Cite this: Med. Chem. Commun., 2014, 5,1208

Received 13th February 2014 Accepted 15th April 2014

DOI: $10.1039 / \mathrm{c} 4 \mathrm{md} 00056 \mathrm{k}$

www.rsc.org/medchemcomm

\section{Response of osteoblast-like MG63 on neoglycosylated collagen matrices}

\author{
Laura Russo, $\uparrow^{\mathrm{a}}$ Antonella Sgambato, $\uparrow^{\mathrm{a}}$ Paolo Giannoni, $\uparrow^{\mathrm{b}}$ Rodolfo Quarto, ${ }^{\mathrm{b}}$ \\ Simone Vesentini, ${ }^{\mathrm{C}}$ Alfonso Gautieri ${ }^{\mathrm{c}}$ and Laura Cipolla*a
}

Biomaterial surface properties, via introduction of specific signal biomolecules can influence cell adhesion and differentiation processes. Inspired by the monosaccharide motifs found in collagen glycosylation patterns, galactose being one of the most commonly found saccharidic residues, collagen matrices were modified in order to expose galactose residues to cells. The interaction of chemically neoglycosylated matrices was evaluated with osteosarcoma-derived cell line MG63.

\section{Introduction}

A variety of biomaterials for regenerative medicine are being developed to support cellular attachment, proliferation and lineage specific differentiation. ${ }^{1}$ Recent advances include bioactivated materials with suitable biomolecules eliciting the desired cellular response toward tissue regeneration. ${ }^{2}$ Collagen is the most commonly used biomaterial and it has found many biomedical applications, such as implants, ${ }^{3,4}$ implant coatings, ${ }^{5}$ scaffolds for artificial organs,,${ }^{6,7}$ grafts, ${ }^{8,9}$ and sutures. ${ }^{10}$ In order to be used as a scaffold for tissue engineering applications, it should allow cell adhesion and proliferation. Nevertheless, given its successful application as a safe and widely accepted biomaterial, ${ }^{11}$ we decided to evaluate the biological behaviour of collagen-based 2D matrices functionalized with glycan moieties that were shown not affecting its structural features, ${ }^{12}$ and reported as having a role in osteoarthritic models, thus requiring in depth studies. ${ }^{13}$ In general, carbohydrates present several interesting features to be considered when linked to material surfaces.

(i) Carbohydrates are polyhydroxylated molecules, hence they are able to increase the hydrophilicity/wettability of the material surface. This parameter is particularly relevant to biomaterial design, since it largely alters the type (and state) of proteins adsorbed from solution, which in turn affects cell adhesion. ${ }^{14}$

(ii) Carbohydrates are signalling molecules. Many studies have focused on engineering the cell-biomaterial interfaces: ${ }^{15}$ an innovative approach is to graft signalling cues (i.e. peptides

\footnotetext{
${ }^{a}$ Dept. of Biotechnology and Biosciences, University of Milano-Bicocca, Piazza della Scienza 2, 20126 Milano, Italy.E-mail: laura.cipolla@unimib.it

${ }^{b}$ Dept. of Experimental Medicine, University of Genova, Via L. B. Alberti 2, 16132 Genova, Italy.E-mail: paolo.giannoni@unige.it; rodolfo.quarto@unige.it

${ }^{c}$ Dept. Elettronica, Informazione e Bioingegneria, Politecnico di Milano, Via Golgi 39, 20133 Milan, Italy

$\dagger$ These authors contributed equally to this work.
}

and carbohydrates) onto surfaces to impart specific biofunctionality. ${ }^{1}$ Carbohydrates coat the surfaces of virtually all mammalian cells determining their interactions with the surroundings. Due to the relevant and still underestimated roles of carbohydrates in mediating biological interactions, ${ }^{16-18}$ they are of paramount relevance for the synthesis and the study of biomaterials grafted with carbohydrate moieties. To date, this approach has been underexploited, and few examples appeared in the literature. ${ }^{19}$ This is probably due to the synthetic challenges that burden carbohydrate modifications.

(iii) As for collagen, glycosylation is emerging as a key issue in determining physiological and pathological states (i.e. osteoarthritis $^{20}$ or ostheogenesis imperfecta). ${ }^{21}$ Evidence is appearing that the glycosylated collagen is deeply involved in remodelling and breakdown of ECM, in the control of the dynamic balance between collagen deposition and turnover. ${ }^{22,23}$ Collagen is usually glycosylated with small glycidic epitopes, $\beta$-galactosides or $\alpha$-(1-2)-glucosyl- $\beta$-galactosides on hydroxylysine residues. Herein we propose the chemical modification of collagen matrices exposing galactose moieties at lysine residues, the comparison, by molecular dynamics simulations, of solvent accessibility of galactose units in native (1, Fig. 1) and in neoglycosylated collagen matrices (2, Fig. 1), and finally the in vitro evaluation of the native and neoglycosylated scaffolds.

Given the relevance of glycosylation on the collagen matrix and the predominant role of collagens in skeletal tissues, it seemed worthwhile to evaluate the interaction of chemically neoglycosylated matrices with MG63 cells, an osteosarcomaderived line that partially mimics the characteristics of human osteoblasts. For these cells collagen-soaked surfaces relevantly implemented cell adhesion. ${ }^{24}$ Moreover MG63 cells were shown to modify their metabolism and gene expression pattern according to the topography of their substrates, ${ }^{25,26}$ ultimately affecting attachment and proliferation. 

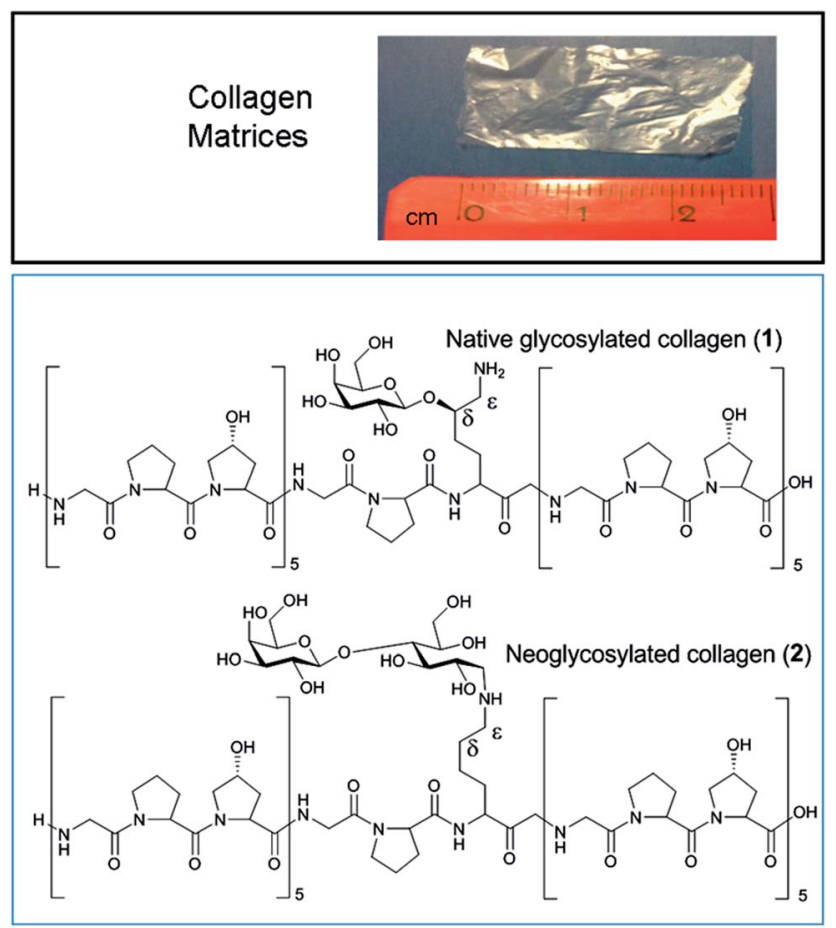

Fig. 1 Top: photograph acquired by a standard camera of the collagen matrices prepared by the solvent-casting method. Bottom: structure of the native glycosylated collagen sequence and the corresponding neoglycosylated collagen used for molecular dynamics simulation (top).

\section{Results and discussion}

\section{Collagen matrix preparation}

Inspired by the monosaccharide motifs found in collagen glycosylation patterns, being galactose one of the saccharidic residues most commonly found, collagen matrices were modified by reductive amination on lysine residues with lactose as a saccharide, affording structure 2 (Fig. 1). ${ }^{12}$ The chemically modified collagen matrices were produced as thin transparent films $\left(1 \mathrm{mg} \mathrm{cm}^{-2}\right)$ (Fig. 1, top image), prepared from collagen type I from an equine tendon as previously described. ${ }^{27}$ The effectiveness of the neoglycosylation procedure can be ascertained in a qualitative way, determining the changes in the hydrophilic character of the matrix surfaces by water contact analysis (WCA). The presence of sugar residues on the material surface, due to their polyhydroxylated nature, should result in an increase in the hydrophilic character of the matrices. Indeed, the WCA changes from $88.38^{\circ} \pm 0.79^{\circ}$ for the native collagen to $69.97^{\circ} \pm 0.80^{\circ}$ for the neoglycosylated samples. Moreover, in order to add quantitative estimation of the neoglycosylation reaction, the effectiveness of the proposed procedure was determined by ${ }^{1} \mathrm{H}$ NMR spectroscopy, using maleic anhydride for the quantification of residual lysine amino groups: roughly, $20 \mathrm{nmol}$ of lactose per $\mathrm{cm}^{2}$ have been added by reductive amination. Regarding the structural assembly of collagen molecules, the untreated collagen matrices observed by tappingmode atomic force microscopy reveals a moderately rough, grainy surface interspersed by long, straight collagen fibrils of various sizes and randomly oriented. ${ }^{12}$ The neoglycosylation reaction does not alter the appearance of the specimen.

\section{Molecular dynamics simulation}

Carbohydrates are able to exploit their biological roles if exposed in an accessible way to their complementary receptors. In order to compare the exposition of galactose units on hydroxylysine residues (1, Fig. 1) and in the adduct obtained by lysine conjugation with lactose (2, Fig. 1), computational studies have been performed by molecular dynamics simulations.

The THeBuScr (Triple-Helical collagen Building Script) code ${ }^{28}$ was used to build a model of the collagen molecule, as previously reported..$^{29,30}$ The simplest model of collagen with only glycine-proline-hydroxyproline (GPO) triplets on each of the three chains in order to represent an archetype of the collagen molecule was used, truncated to 15 amino acids per chain, $\left[(\mathrm{GPO})_{11}\right]_{3}$, due to computational limitations, since the full length collagen molecule (300 $\mathrm{nm}$ long) is too large for computational simulations. Peptides of comparable length have been used both in earlier computational ${ }^{29,31,32}$ and experimental studies. ${ }^{33}$ In one of the three chains, the proline residue in the central triplet (the sixth triplet) is substituted by hydroxylysine or lysine, which represents the glycosylation site. Molecular dynamics simulations are performed to assess the equilibrium configuration of the native and neoglycosylated collagen sequences 1 and 2 (Fig. 1).

In both cases the sugar ring is well exposed and highly solvated (Fig. 2). In order to quantitatively assess the interaction of the two glycidic moieties with the surrounding solvent the average number of $\mathrm{H}$-bonds between the saccharidic residues and water was calculated. The results show that the native
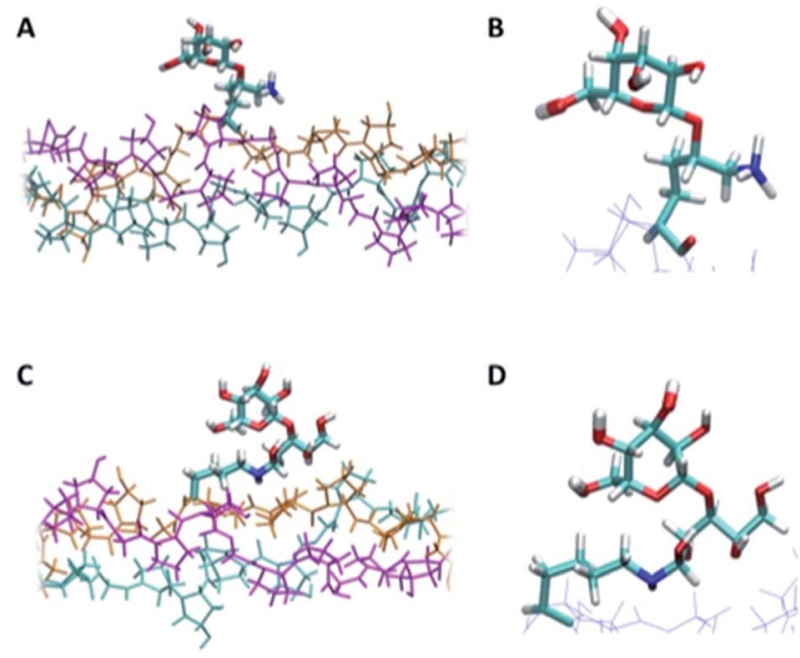

Fig. 2 Equilibrium structure of $O$-glycosylated collagen hydroxylysine (panel A, zoom in panel B) and neoglycosylated collagen (panel C, zoom in panel D). Hydroxyl groups at position 3, 4, 6 are highlighted in the zoomed images. The explicit solvent is not shown for the sake of clarity. 
sequence forms $4.35 \pm 1.78 \mathrm{H}$-bonds whereas the neoglycosylated collagen forms $6.09 \pm 1.93$. The slightly larger number of $\mathrm{H}$-bonds found for the neoglycosylated collagen can be explained taking into account the greater number of $-\mathrm{OH}$ groups available for hydrogen bonding with water. In the native $O$-glycosylated collagen the terminal $\mathrm{NH}_{3}{ }^{+}$group of lysine is preserved, partially compensating the lower number of hydroxyl groups.

The H-bonds with particular reference to hydroxyl groups at carbon 3, 4 and 6 of the galactose unit were also analyzed, since they are putative interacting sites with their corresponding receptors (i.e. galectins). ${ }^{34}$ The galactose on hydroxylysine (native form) shows an average of $0.69,0.47$ and $0.36 \mathrm{H}$-bonds, respectively, at position 3, 4 and 6 (Fig. 2, panel B). On the other hand, the hydroxyl groups of the neoglycosylated collagen forms $0.49,0.48$ and $0.30 \mathrm{H}$-bonds (respectively at position 3,4 and 6, Fig. 2, panel D). The molecular dynamic simulation suggests that the sugar rings in the native and neoglycosylated forms are very similarly accessible to their receptor.

\section{Biological evaluation}

A rapid and extended colonization of all available surfaces and volumes of a suitable substrate by progenitor cells should be a prerequisite to maximize the regenerative effects of a cellloaded scaffold within a lesion site. In this respect, matrices able to drive and sustain a large proliferative burst may be beneficial, provided that they do not influence the differentiation potential of the cells. For bone tissue engineering it is also relevant to note that proper glycosylation of collagen $\mathrm{I}$ is a prerequisite for the development of a suitable vessel network: angiogenesis does not occur if endothelial cells are grown on substrates derived from de-glycosylated collagen matrices or derived from normal fibroblasts. In contrast it becomes markedly sustained in over-glycosylated collagen I matrices derived from Osteogenesis imperfecta mutated fibroblasts. ${ }^{35}$ Thus, a substrate providing such a pro-angiogenic signal may indeed be advantageous. Given these premises, the biological assays with the human osteosarcoma MG63 cell line were performed to determine: (i) if the chosen grafting strategies could be applied to obtain sterile collagen matrices suitable for further cell seeding and culturing procedures; (ii) if glycosylated matrices exerted any direct effect on the attachment and proliferation of this osteoblast-like cell line. Indeed, on over 20 independent experiments on native and neoglycosylated collagen as cell culture platforms (for a total of 40), we did not notice any statistical variation in the number of adhered cells after seeding, nor did we experience any contamination. However, the proliferation rates of the attached cells were quite different on the two collagen matrices. As shown in Fig. 3 panel A, control cells grown on plastic or on glycosylated collagen performed 3.8-4.0 doublings within the experimental timing (cell duplication time: $42 \mathrm{~h}$ ), while cells grown on the native collagen performed less than 3.0 doublings (cell duplication time: $58 \mathrm{~h}$ ). These results are in agreement with the images of the cellseeded matrices acquired at the end of the experimental timings (Fig. 3, panel B).
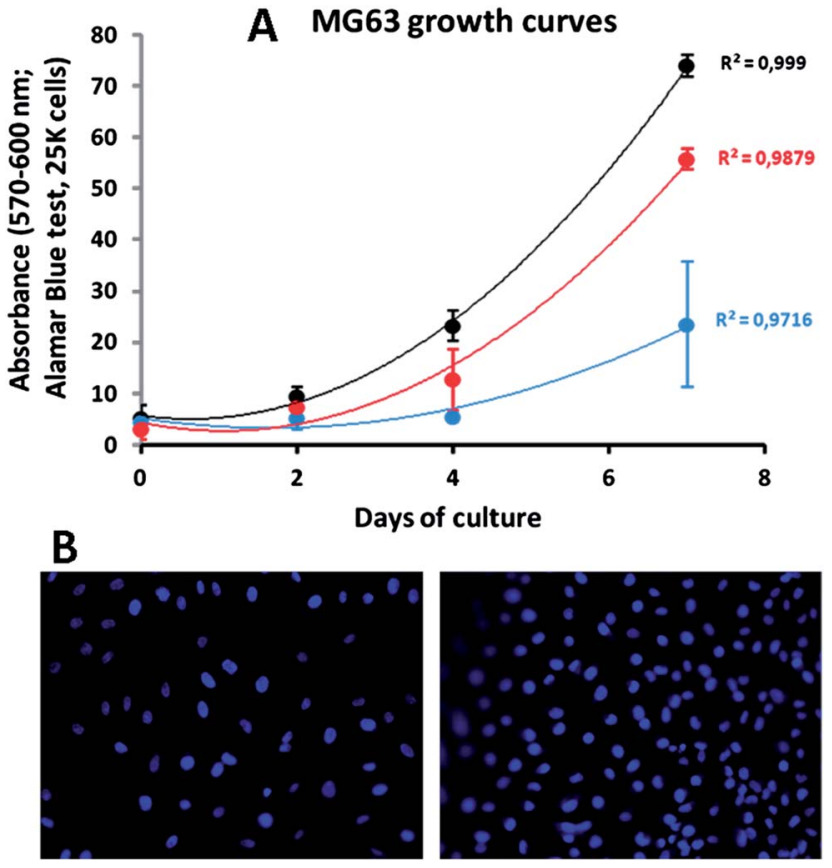

Fig. 3 (A) MG63 growth rate on native and neoglycosylated collagen: $(\bullet)$ standard plastic; (๑) control collagen; (๑) glycosylated collagen; ( $\rightarrow$ best-fit standard plastic; (-) best-fit control collagen; (-) best-fit glycosylated collagen. Data points depict mean values \pm SD of 6 independent determinations. (B) Fluorescence images of DAPI-stained MG63 nuclei after cells were grown for 7 days onto native or neoglycosylated collagen substrata, the left and right panel, respectively (images are representative of five different visual fields acquired for each substratum; $20 \times$ enlargements).

\section{Conclusions}

Carbohydrates play relevant roles in cell-matrix interactions ${ }^{36}$ that are still far from a profound comprehension; in this work we proposed the study of chemically modified collagen matrices exposing galactose moieties and their preliminary in vitro evaluation with selected cell lines, in order to gain insight on the cell responses to the grafted carbohydrates. As a whole, the presented results evidenced that the neoglycosylated collagen can be recognized as a preferential substrate for the growth of cells of the skeletal system. Prospectively, this chemical modification could be used to implement cell colonization of collagen-based scaffolds for tissue engineering approaches.

\section{Experimental}

\section{Molecular dynamics simulation}

Explicit water molecular dynamics simulations are performed using the LAMMPS code $^{37}$ and the REAX force field, ${ }^{38}$ which include partial charge calculations using the QEq method. Since the collagen triple helix considered here is truncated, the $\mathrm{N}$-terminal is capped with an acetyl group, whereas the $\mathrm{C}$ terminal with the corresponding amide. The collagen peptides are solvated in a water box, and ions are included to achieve an ionic concentration of $0.15 \mathrm{~mol} \mathrm{~L}^{-1}$. The total number of atoms of the solvated system is approximately 25000 atoms. 
Nonbonding interactions are computed using a cut-off for a neighbour list at $1.35 \mathrm{~nm}$, with a switching function between 1.0 $\mathrm{nm}$ and $1.2 \mathrm{~nm}$. The preliminary energy minimization is performed by using a conjugate gradient algorithm until convergence. The systems are then equilibrated at a temperature of $310 \mathrm{~K}\left(37^{\circ} \mathrm{C}\right)$ for $100 \mathrm{ps}$ of molecular dynamics. We observe that the root mean square deviation (RMSD) of the protein reaches a stable value within $\approx 100$ ps simulation time, thus we assume that the collagen peptides are equilibrated properly. We use Visual Molecular Dynamics (VMD) ${ }^{39}$ for analysis and rendering. Hydrogen bonds (H-bonds) are calculated on a geometric basis using a cut-off of $3.5 \AA$ and $25^{\circ}$ and are mediated over the stable portion.

\section{Collagen matrix preparation}

Collagen matrices. Type I collagen films from equine tendon (1\% collagen gel in acetic acid) were produced by the solventcasting method. ${ }^{27}$ After dilution $1: 6 \mathrm{w} / \mathrm{v}$ in ultrapure water of the $1 \%$ gel, the suspension was homogenized at $4{ }^{\circ} \mathrm{C}$ with a mixer for 2 minutes at maximum speed. After removal of the aggregates, $40 \mathrm{~mL}$ of collagen solution was poured into an $8.5 \times$ $12.5 \mathrm{~cm}$ culture multiwell lid and the solvent evaporated in the fume hood for two days.

Neoglycosylation. A piece of a collagen matrix $(80 \mathrm{mg}, 12 \mathrm{~cm}$ $\times 7 \mathrm{~cm})$ was immersed in $20 \mathrm{~mL}$ of lactose aq. solution $(0.06 \mathrm{M})$ followed by the sequential addition of $0.03 \mathrm{M}$ of $\mathrm{NaCNBH}_{3}$ in citrate buffer ( $\mathrm{pH}$ 6.0) and reacted overnight. After some time the collagen film was thoroughly washed first with $20 \mathrm{~mL}$ of $\mathrm{mQ}$ $\mathrm{H}_{2} \mathrm{O}$ three times for 20 minutes, and finally with ethanol (20 $\mathrm{mL}$ ) for 20 minutes.

\section{NMR analysis}

Derivatization of the native and neoglycosylated collagen samples with maleic anhydride was performed to label and quantify $-\mathrm{NH}_{2}$ groups of lysine residues. Collagen films (32 mg) were immersed in a THF solution $(0.04 \mathrm{M})$ of maleic anhydride in the presence of $\mathrm{NaHCO}_{3}$. The reaction was carried out at room temperature overnight. After thoroughly washing with mQ $\mathrm{H}_{2} \mathrm{O}$, followed by ethanol, collagen films were dried under vacuum and then dissolved in $0.6 \mathrm{~mL}$ of $2 \mathrm{M} \mathrm{NaOD}$ in $\mathrm{D}_{2} \mathrm{O} .{ }^{1} \mathrm{H}$ NMR spectra were recorded using a Varian $400 \mathrm{MHz}$ Mercury instrument, operating at a proton frequency equal to $400 \mathrm{MHz}$, at room temperature. The $90^{\circ}$ pulse-width (pw90) was calibrated; the number of scans varied depending on the signal-tonoise ratio, and the recycling delay was $5 \mathrm{~s}$.

\section{Cell culturing and proliferation assay}

Human osteosarcoma MG63 cells were cultured in Coon's modified F12 Medium (F12; Biochrom AG, Berlin, Germany) containing $10 \%$ foetal calf serum (FCS; GIBCO Invitrogen Corp., Milano, Italy) at $37{ }^{\circ} \mathrm{C}$ and $5 \% \mathrm{CO}_{2}$. The control and chemically modified collagen matrices, layered onto support polystyrene plates, were deposited into the wells of 24-well plates, previously coated with a sterile $1 \%$ agarose solution to avoid cell attachment and growth outside the matrices. Cells were then seeded onto the matrices at a density of $25 \times 10^{4}$ cells per well and let adhere for $4 \mathrm{~h}$. Alternatively, control cultures were performed on the untreated standard plastic ware; for each culture condition six independent replica wells were prepared. Unattached cells were removed and each well was washed twice with sterile phosphate buffered solution, pH 7.4 (PBS). A fresh medium, supplemented with $10 \%$ Alamar Blue ${ }^{\mathrm{TM}}$ (Life Science Invitrogen, Milano, Italy) was added and the plates were incubated at $37^{\circ} \mathrm{C}$ in the dark for the subsequent $4 \mathrm{~h}$. Supernatants were collected and briefly centrifuged (14000 rpm, $30 \mathrm{~s}$ ) while each well was replenished with fresh $\mathrm{F} 12+10 \%$ FCS. This procedure was repeated after 2,4 and 7 days of culture. To assess cell growth in each culture condition at each time point, equal volumes of supernatants $(200 \mu \mathrm{L})$ were assessed in duplicates, according to the manufacturer's instructions, at 570 and 600 nm using a Spectra MR spectrophotometer (Dynex Technologies, Chantilly, VA, USA). After 7 days of culture, matrix specimens were washed twice in PBS, carefully removed from the polystyrene plates and deposited onto a glass slide. Matrices were then fixed in $4 \%$ formaldehyde in PBS for $30 \mathrm{~min}$ at RT and subsequently exposed to a $3 \mu \mathrm{M}$ solution of DAPI (Invitrogen Molecular Probes; Milano, Italy) in PBS for 15 min. Stained matrices were then re-washed in PBS, layered with a drop of Dako Fluorescent mounting medium (Dako North America Inc., Carpinteria, CA, USA) and covered with a cover slip glass. Images were acquired using a Nikon Digital Sight DS-5Mc camera mounted on an Olympus BX51 fluorescence microscope.

\section{Acknowledgements}

This work has been supported by Fondazione Cariplo, Grant no. 2010-0378, and Grant no. 2011-0270, MIUR under project PRIN 2010L9SH3K.

\section{Notes and references}

1 M. D. Mager, V. LaPointe and M. M. Stevens, Nat. Chem., 2011, 3, 582-589.

2 L. Cipolla, L. Russo, N. Shaikh and F. Nicotra, Materials biofunctionalization for tissue regeneration, CRC Press, 2013.

3 A. J. Bailey, Wound Repair Regen., 2000, 8, 5-12.

4 N. J. Smart and S. Bloor, Surg. Innovat., 2012, 19, 221-229.

5 See for example: M. Vandrovcová, T. Douglas, D. Hauk, B. Grössner-Schreiber, J. Wiltfang, L. Bačáková and P. H. Warnke, Physiol. Res., 2011, 60, 797-813.

6 I. V. Yannas, D. S. Tzeranis, B. A. Harley and P. T. So, Philos. Trans. R. Soc., A, 2010, 368, 2123-2139.

7 L. Hench and J. R. Jones, Biomaterials, artificial organs and tissue engineering, Imperial College of Science, Technology and Medicine, 2005.

8 W. He, T. Yong, W. E. Teo, Z. Ma and S. Ramakrishna, Tissue Eng., 2005, 11, 1574-1588.

9 F. Boccafoschi, J. Habermehl, S. Vesentini and D. Mantovani, Biomaterials, 2005, 2635, 7410-7417.

10 T. Okada, T. Hayashi and Y. Ikada, Biomaterials, 1992, 13, 448-454. 
11 E. A. Abou Neel, L. Bozec, J. C. Knowles, O. Syed, V. Mudera, R. Day and J. Keun Hyun, Adv. Drug Delivery Rev., 2013, 65, 429-456.

12 L. Russo, A. Gautieri, M. Raspanti, F. Taraballi, F. Nicotra, S. Vesentini and L. Cipolla, Carbohydr. Res., 2014, 389, 12-17.

13 L. Russo, C. Battocchio, V. Secchi, E. Magnano, S. Nappini, F. Taraballi, L. Gabrielli, F. Comelli, A. Papagni, B. Costa, G. Polzonetti, F. Nicotra, A. Natalello, S. M. Doglia and L. Cipolla, Langmuir, 2014, 30, 1336-1342.

14 A. S. G. Curtis, J. Cell Sci., 1984, 71, 17-35.

15 M. M. Stevens and J. H. George, Science, 2005, 310, 11351138.

16 K. W. Moremen, M. Tiemeyer and A. V. Nairn, Nat. Rev. Mol. Cell Biol., 2012, 13, 448-462.

17 K. Ohtsubo and J. D. Marth, Cell, 2006, 126, 855-867.

18 A. Franco, D. A. Tilly, I. Gramaglia, M. Croft, L. Cipolla, M. Meldal and H. M. Grey, Nat. Immunol., 2000, 1, 145-150.

19 L. Cipolla, L. Russo, F. Taraballi, C. Lupo, D. Bini, L. Gabrielli, A. Capitoli and F. Nicotra, Smart biomaterials: the contribution of glycoscience, in Specialist Periodical Reports, SPR Carbohydrate Chemistry, 2012, vol. 38, ch. 17, pp. 416-445, ISBN: 9781849734394.

20 B. Dzhambazov, M. Holmdahl, H. Yamada, S. Lu, M. Vestberg, B. Holm, O. Johnell, J. Kihlberg and R. Holmdahl, Eur. J. Immunol., 2005, 35, 357-366.

21 Y. Taga, M. Kusubata, K. Ogawa-Goto and S. Hattori, J. Proteome Res., 2013, 12, 2225-2232.

22 H. J. Jurgensen, D. H. Madsen, S. Ingvarsen, M. C. Melander, H. Gårdsvoll, L. Patthy, L. H. Engelholm and N. Behrendt, J. Biol. Chem., 2011, 286, 32736-32748.

23 A. Oikarinen, H. Anttinen and K. I. Kivirikko, Biochem. J., 1976, 156, 545-551.
24 Q. Q. Wang, W. Li and B. C. Yang, J. Biomed. Mater. Res., 2011, 99, 125-134.

25 O. Zinger, K. Anselme, A. Denzer, P. Habersetzer, M. Wieland, J. Jeanfils, P. Hardouin and D. Landolt, Biomaterials, 2004, 25, 2695-2711.

26 S. W. Tsai, H. M. Liou, C. J. Lin, K. L. Kuo, Y. S. Hung, Y. S. Weng and F. Y. Hsu, PLoS One, 2012, 7, e31200.

27 F. Taraballi, S. Zanini, C. Lupo, S. Panseri, C. Cunha, C. Riccardi, M. Marcacci, M. Campione and L. Cipolla, J. Colloid Interface Sci., 2013, 394, 590-597.

28 J. Rainey and M. Goh, Bioinformatics, 2004, 20, 2458-2459.

29 A. Gautieri, M. I. Pate, S. Vesentini, A. Redaelli and M. J. Buehler, J. Biomech., 2012, 45, 2079-2083.

30 Z. Qin, A. Gautieri, A. K. Nair, H. Inbar and M. J. Buehler, Langmuir, 2012, 28, 1982-1992.

31 A. Gautieri, S. Vesentini, A. Redaelli and R. Ballarini, Int. J. Nonlinear Mech., 2013, 56, 25-33.

32 P. J. Veld and M. J. Stevens, Biophys. J., 2008, 95, 33-39.

33 A. Persikov, J. Ramshaw, A. Kirkpatrick and B. Brodsky, Biochemistry, 2000, 39, 14960-14967.

34 S. Kumar, M. Frank and R. Schwartz-Albie, PLoS One, 2013, 8, e59761.

35 D. Palmieri, M. Valli, S. Viglio, N. Ferrari, B. Ledda, C. Volta and P. Manduca, Exp. Cell Res., 2010, 316, 789-799.

36 L. Russo, A. Sgambato, M. Lecchi, V. Pastori, M. Raspanti, A. Natalello, S. M. Doglia, F. Nicotra and L. Cipolla, ACS Chem. Neurosci., 2014, 5, 261-265.

37 S. Plimpton, J. Comput. Phys., 1995, 117, 1-19.

38 A. C. T. Duin, S. Dasgupta, F. Lorant and W. A. Goddard, J. Phys. Chem. A, 2001, 105, 9396-9409.

39 W. Humphrey, A. Dalke and K. Schulten, J. Mol. Graphics, 1996, 14, 33-38. 